\title{
La Transition politique en Espagne
}

Dolores Avilés de Torres

Traducteur : Claudine Adam

\section{(2)enEdition}

\section{Journals}

Édition électronique

URL : http://journals.openedition.org/ries/3252

DOI : 10.4000/ries.3252

ISSN : 2261-4265

Éditeur

Centre international d'études pédagogiques

Édition imprimée

Date de publication : 1 mars 1997

Pagination : 93-105

ISSN : 1254-4590

Référence électronique

Dolores Avilés de Torres, «La Transition politique en Espagne », Revue internationale d'éducation de Sèvres [En ligne], 13 | 1997, mis en ligne le 24 juillet 2013, consulté le 19 avril 2019. URL : http:// journals.openedition.org/ries/3252 ; DOI : 10.4000/ries.3252 


\section{La Transition politique en Espagne ${ }^{1}$}

Dolores Avilés de Torres

En Espagne, le changement politique entraîné par le passage de la dictature du général Franco, décédé en 1975, à l'État démocratique défini par la Constitution de 1978, n'a pas été le résultat d'une « rupture » politique soudaine ni rapide, mais d'un processus plus lent qui avait déjà commencé du vivant du dictateur. Lorsque le changement a commencé à devenir perceptible, les historiens espagnols ont d'ailleurs respecté le nom que la société espagnole avait forgé : la «Transition ». Transition graduelle, avec des rythmes d'accélération différents.

Quelles sont les limites chronologiques de la Transition espagnole? Les historiens ne sont pas tous d'accord: pour ceux dont les critères sont strictement politiques, on devrait en fixer le début en 1974, premier moment, où pour raison de santé, Franco fut obligé de céder provisoirement ses pouvoirs au prince d'Espagne, aujourd'hui, le roi Juan Carlos $1^{\text {er }}$ et où, dans le même temps, des forces importantes de l'opposition créèrent la Junte démocratique dans le but de favoriser un changement politique ; ils établissent la fin de la Transition en 1978, année durant laquelle fut approuvée la Constitution démocratique en vigueur aujourd'hui.

D'autres historiens proposent une période de temps plus longue, car ils tiennent compte aussi de critères sociaux et culturels : du début des années soixante-dix, quand l'opposition intérieure a déjà une large base sociale et se manifeste déjà dans certains faits quotidiens, jusqu'en 1982, année de la première victoire électorale du Parti socialiste ouvrier espagnol, dont la signification historique dépasse le contenu de cet article.

Quelles limites chronologiques choisirons-nous ici ? Celles qui s'harmoniseront le mieux avec notre sujet, l'enseignement de l'histoire : du début des années soixante-dix, lorsque la Transition sociale et culturelle commence déjà à se faire sentir dans de nombreux établissements scolaires, jusqu'à l'approbation par référendum de la Constitution de 1978, grâce à laquelle la liberté d'expression, de recherche, d'enseignement universitaire et d'enseignement en général est établie et garantie.

Il nous faut avoir conscience qu'un grand laps de temps a passé depuis lors : dix-huit ans. Nous ne parlons ni d'un passé immédiat comme c'est le cas pour les pays d'Europe de l'Est, ni aussi lointain que celui de l'Allemagne ou du

1 Cet article a été traduit par Claudine Adam. 
Japon. C'est pourquoi, il me semble nécessaire de ne pas me limiter à une époque qui se terminerait en 1978, mais de faire référence aussi aux années postérieures. C'est seulement ainsi que cet article atteindra son objectif.

Dernière remarque : cet article est basé sur mon expérience personnelle, sur ma propre réflexion à partir de ce que j'ai vécu et connu en tant qu'étudiante en histoire à l'université Complutense de Madrid dans les années soixante, et en tant que professeur dans le secondaire, dans des établissements privés et publics à partir de 1967, c'est-à-dire pendant les dernières années de la dictature, pendant la Transition et toutes les années qui ont suivi jusqu'à maintenant. Dans cet article, je me référerai donc presque exclusivement au niveau du secondaire; mais ce que j'explique est aussi valable, logiquement, pour l'enseignement de l'histoire dans les autres niveaux non universitaires. Je m'appuie aussi sur les observations que j'ai faites et les expériences que j'ai vécues lorsque je fis partie du comité de direction du collège des Licenciados ${ }^{2}$ de 1973 à 1977, ce qui m’a permis de voir, sous un angle très intéressant, comment le monde de l'enseignement a vécu ces années-là.

\section{Le cadre législatif : la loi générale d'éducation}

Ce n'est absolument pas un hasard si la Transition espagnole coïncide avec l'implantation graduelle d'une des lois espagnoles les plus importantes en matière d'éducation. Loi qui a voulu changer le système d'enseignement et ses contenus, mais aussi ses formes et ses méthodes : la loi générale d'éducation de 1970, encore en vigueur jusqu'à la complète implantation de la loi d'aménagement général du système éducatif (LOGSE), approuvée en 1990, qui la remplace.

La loi générale d'éducation instaurait une étape d'enseignement obligatoire et gratuite, d'une durée de huit ans, pour tous les enfants espagnols de six à quatorze ans, (enseignement général de base - EGB), suivie d'un cycle de bachillerato en trois ans (bachillerato unifié polyvalent - BUP) ou d'une formation professionnelle de deux cycles. L'histoire figurait dans les trois dernières années de l'EGB, à l'intérieur des sciences sociales, dans deux années du BUP (histoire des civilisations, géographie et histoire de l'Espagne), et dans le premier cycle de la formation professionnelle (matières générales).

En elle-même, la loi générale d'éducation impliquait une synthèse et une reconnaissance des profonds changements qui s'étaient produits et continuaient à se produire au sein de la société espagnole depuis les années soixante. Parmi les principes que l'on voulait implanter comme valeurs du nouveau système éducatif, Manuel de Puelles Benítez souligne : l'égalité des chances, les relations entre enseignement et travail, l'ouverture pédagogique, la préoccupa-

2 Titre universitaire équivalent en France à bac +5 . 
tion pour la qualité de l'enseignement, la réforme des programmes, la réforme des contenus de l'enseignement, l'autonomie des établissements, l'innovation pédagogique ${ }^{3}$... Principes qui seront fortement en contradiction avec le cadre politique dans lequel devait s'implanter cette loi.

Cette loi ne doit pas être interprétée comme un désir de démocratisation du régime franquiste, mais comme un des résultats de l'épuisement intérieur du régime et un manque de capacité à résoudre ses propres contradictions. Avec le recul des vingt années qui se sont écoulées depuis son approbation, on parle peu du fait qu'elle implique et synthétise l'échec de plus de trente années de franquisme en matière d'éducation. "La déclaration d'intentions de la loi répond à notre tradition libérale, ce qui représente aussi tacitement une déclaration voilée de trente ans d'éducation autoritaire ${ }^{4}$.»

La loi générale d'éducation naquit donc avec la Transition, s'implanta en même temps que celle-ci se développait et, transformée par la Constitution de 1978, elle indiqua les contenus, les orientations et la méthodologie de l'enseignement de l'histoire. Durant les premières années de la Transition et de l'implantation de cette loi, pourtant, les nouveautés qu'elle impliquait durent coexister dans les établissements avec les contenus et les orientations des anciennes lois d'enseignement, elles aussi franquistes, qui disparaissaient.

Quelles nouvelles orientations établissait la LGE pour l'enseignement de l'histoire? Quelle différence y avait-il entre ces dernières et celles établies durant la dictature ? Comment les unes et les autres se concrétisaient-elles dans les manuels? Et, surtout, comment étaient-elles interprétées, comment se comportaient les professeurs dans la réalité quotidienne de leur salle de classe?

Pour mieux comprendre la valeur du changement instauré durant la Transition dans l'enseignement de l'histoire, il nous faut absolument comparer les contenus et les orientations marqués par les lois antérieures de Franco avec les nouveautés introduites sur ce terrain par la loi générale de l'éducation.

\section{L'enseignement de l'histoire de 1936 au début de la Transition}

\section{Contenus et orientations}

Quelle était l'importance, quelle était la signification de l'enseignement de l'histoire de l'Espagne et de l'histoire universelle pour le franquisme? Nous reproduisons ici quelques paragraphes du préambule de la loi de 1938 sur la réforme de l'enseignement secondaire. On y trouve condensée l'interprétation

3 M. de Puelles Benitez, Éducation et idéologie dans l'Espagne contemporaine (1967-1975), Barcelone, Éd. Labor, 1980, p. 428.

4 Ibid., p. 426. 
que l'on voulait transmettre de ces deux domaines aux «futures classes dirigeantes ».

En évoquant la culture classique et les "humanités » dans la formation des futurs bacheliers, elle considère que ces matières sont « le plus sûr chemin pour la revalorisation de l'essence authentique de l'Espagne, de l'Espagne formée dans les études classiques et humanistes de notre Xvie siècle, qui produisit cette pléiade d'hommes politiques et de guerriers (...) de notre époque impériale, vers laquelle retourne la vocation héroïque de notre jeunesse; pouvoir de formation encore remarquablement corroboré par l'exemple des grandes nations impériales modernes..." (cela se rapportait sans doute à l'Allemagne nazie et à l'Italie fasciste). La définition qui est faite de "l'essence authentique de l'Espagne» est complétée par cet autre paragraphe du même préambule : « (...) le catholicisme est au cœur de l'histoire de l'Espagne (...). La revalorisation de ce qui est espagnol, l'extirpation définitive du pessimisme antihispanique venant de l'étranger, fils de l'apostasie et de l'odieuse et mensongère légende noire, doivent être obtenues grâce à l'enseignement de l'histoire universelle (...). Il s'agit ainsi de mettre en évidence la pureté de la nationalité espagnole, la catégorie supérieure, universaliste de notre esprit impérial, de l'hispanité (...), défenseur et missionnaire de la vraie civilisation, qui est la chrétienté ${ }^{5}$.»

Dans cette loi apparaissent déjà clairement les orientations qui guideront l'enseignement de l'histoire pendant plusieurs années : un sentiment nationaliste exacerbé ; une interprétation biaisée de ce nationalisme, qui identifie l'Espagne "authentique» avec l'époque la plus brillante de l'Empire, le XVIe siècle; un fort intégrisme religieux; un rejet sur l'étranger de la responsabilité de tout ce qui ne suivra pas cette ligne - la décadence de l'Espagne a des causes qui lui sont étrangères et extérieures: l'irréligiosité ou l'hostilité des autres nations (la « légende noire ») ; les aspirations impérialistes, même si elles étaient formulées de façon vague et confuse en raison de motifs politiques du moment, dont l'explication dépasse le cadre de cet article.

Pendant le franquisme, les enseignements de l'histoire de l'Espagne et de l'histoire universelle étaient au service de l'idéologie du "Nouvel État » et leur objectif primordial était donc l'endoctrinement des nouvelles générations.

Il ne faut pas croire que de telles orientations impliquaient une grande nouveauté. Il s'agissait plutôt de la continuation d'un enseignement ayant des contenus et des orientations à caractère extrêmement conservateur et intégriste issu d'une époque très antérieure à la dictature de Franco. Par contre la nouveauté, c'est l'accent nationaliste prononcé, de caractère phalangiste conséquence indubitable de la présence de la Phalange espagnole dans la formulation

5 Cité dans le même ouvrage, p. 371 et 372. 
des bases idéologiques du « Nouvel État» élaborées par le franquisme débutant, et qui plus tard seront appelées par les historiens « national-catholicisme ».

A ces orientations de l'enseignement de l'histoire que nous avons extraites de la loi de 1938, il faudrait en ajouter une autre, et non des moindres précisément: l'identification de «l'Espagne authentique» avec une Espagne unie. Mais, pour le franquisme, « unie » signifiait le choix d'un système unitaire d'État, fortement centralisé. Toute tentative de décentralisation, d'autonomie ou d'aspiration fédérale revenait à attenter à «l'essence »» de l'Espagne. Cela entrait bien évidemment en contradiction avec la réalité confédérale de l'Espagne « authentique » du XVI ${ }^{\mathrm{e}}$ siècle, contradiction évitée par l'interprétation franquiste de l'histoire en s'identifiant à la volonté unitaire et centraliste des Habsbourg espagnols, si souvent confrontés à cette réalité confédérale, et en identifiant cette volonté à la réalisation de "la véritable essence » de l'Espagne.

Mais tel n'est pas exactement le sens de l'histoire que l'on voudra changer et que l'on changera pendant la Transition. La défaite des systèmes fascistes et le triomphe des démocrates-libéraux à la fin de la Seconde Guerre mondiale obligeront le régime de Franco à s'adapter, dans le domaine du possible, aux temps nouveaux. En ce qui concerne l'enseignement, la doctrine nationale-catholique franquiste - et l'interprétation de l'histoire qui en découlait - accentua de plus en plus son caractère catholique tandis que le radicalisme nationaliste à caractère phalangiste devenait plus modéré. L'objectif d'endoctrinement qui avait été assigné à l'enseignement de l'histoire perdit de son importance, de même que la caractéristique d' " extérieur à l'essence de l'Espagne » et d' « étranger " pour tout ce qui avait provoqué sa décadence. Mais on ne supprima jamais un autre objectif: celui de justifier la nécessité du régime imposé.

\section{Les manuels scolaires et les professeurs}

Comment de tels contenus étaient-ils enseignés, comment de telles orientations étaient-elles concrétisées, à travers quoi l'histoire était-elle manipulée? Rien de tout cela n'était bien difficile. Le seul matériel didactique sur lequel s'appuyait la presque totalité des professeurs consistait dans les manuels scolaires. Manuels scolaires qui ne pouvaient être publiés sans l'autorisation du ministère de l'Éducation. Manuels scolaires où les auteurs cachaient les faits, exaltaient ou minimisaient les phénomènes, déguisaient des jugements implicites ou utilisaient d'insolents jugements explicites. Il était très difficile à l'étudiant, sinon impossible, de comparer les exposés de son livre d'histoire avec ceux d'autres ouvrages du fait du contrôle exercé sur l'édition.

Les professeurs des années quarante, cinquante et d'une bonne partie des années soixante n'étaient pas non plus en mesure d'enseigner dans les classes d'autres contenus ou de donner d'autres orientations. L'épuration des enseignants au cours des premières années du franquisme, la poursuite des 
études dans des écoles et des universités dominées par le national-catholicisme, l'absence de toute formation autre qu'officielle et, souvent, la crainte ou la prudence, faisaient des professeurs un instrument docile.

\section{L'enseignement de l'histoire pendant la Transition}

\section{Orientations différentes, livres différents, professeurs différents}

Bien qu'à la fin des années soixante on commençât déjà à remarquer des signes indéniables de changement, cette même loi générale d'éducation chercha à assigner d'autres directions au niveau des objectifs et des orientations de l'enseignement de l'histoire. Une des dispositions complémentaires à cette loi ${ }^{6}$, fixant les nouveaux programmes du bachillerato, établit ainsi les objectifs des sciences sociales et anthropologiques, dont faisait partie l'histoire :

«Les sciences sociales et anthropologiques (...) ont pour but d'offrir une large connaissance de l'homme et de la société, en plaçant les élèves devant les faits humains, individuels et collectifs, du passé et du présent. Elles leur apporteront des connaissances et développeront des habitudes qui leur permettent de comprendre en profondeur la culture de la société dans laquelle ils vivent, de contribuer à son amélioration et à son développement et de faciliter la vie en commun et la collaboration avec les autres, l'entraînement aux activités et aux responsabilités civiques et sociales et la compréhension entre les hommes et les peuples. "

Il y a un abîme entre ce paragraphe d'un décret signé par le général Franco quelques mois avant sa mort et ceux de la loi de 1938. Mais ne nous leurrons pas: le cadre politique et l'idéologie officielle toujours en vigueur étaient en totale contradiction avec l'esprit de tels objectifs; de ce fait, ils n'avaient aucune portée ou presque. Les manuels scolaires publiés au fur et à mesure de l'implantation de la LGE, en pleine Transition, répondaient aux nouveaux besoins du bachillerato, même s'ils devaient être obligatoirement approuvés par le ministère de l'Éducation; ils offraient une objectivité et une multitude de critères impensables dix ans auparavant. Mais ces nouvelles qualités ne surprenaient ni les professeurs ni les élèves : la réalité de nombre de classes avait anticipé le changement des contenus et les orientations de l'enseignement de l'histoire.

On manque de travaux de recherche sur l'enseignement de l'histoire au cours de la Transition. C'est pourquoi, ce qui suit est basé sur mon appréciation des faits et mon expérience personnelle.

6 Décret 160/1975 du 23 janvier (BOE du 13 février 1975). 
A mon avis, la plupart des professeurs d'histoire continuaient à baser leurs cours sur les manuels scolaires, anciens ou modernes, sans introduire de changements dans leurs explications sur les faits, les phénomènes ou les jugements qui y étaient exposés, par affinité avec l'idéologie du régime franquiste ou par prudence. Mais une minorité de professeurs, surtout ceux qui avaient terminé leurs études universitaires à partir de la fin des années soixante, rapportaient de l'université d'autres idées et d'autres expériences. Cette minorité s'élargit peu à peu en même temps que se multipliait le nombre de licenciados universitaires et qu'augmentait le nombre de constructions scolaires, conséquence de la pression sociale et des recommandations de la LGE elle-même.

\section{Contenus différents}

En quoi l'histoire enseignée par cette minorité croissante était-elle différente de l'histoire officielle des dernières années du franquisme, déjà très modérée par rapport aux premières années? On peut ainsi résumer les différences selon les points suivants.

Par rapport au passé immédiat, on note une délégitimisation du régime de Franco, aussi bien en fonction de ses origines que de ses modes de fonctionnement.

Par rapport au passé plus lointain, se développe une révision critique des "vérités historiques officielles", des points de vue et des interprétations forgées par l'idéologie officielle dans le but d'identifier ses origines et son caractère avec des faits, des personnages, des processus et des étapes de l'histoire de l'Espagne, en les détournant. C'est-à-dire :

- une démystification des personnages historiques importants exaltés par le franquisme en tant que champions de "l'Espagne authentique » (Isabelle la Catholique, Philippe II...);

- une revendication du caractère espagnol de ce qui avait été rejeté par l'idéologie franquiste dans le passé lointain et proche : personnages historiques, minorités ou peuples entiers. Dans ce sens, revaloriser Al Andalus, l'Espagne musulmane, et revendiquer son caractère espagnol - tout aussi espagnol que celui des royaumes chrétiens qui avaient fini par la vaincre - revenait à s'attaquer à un des mensonges les plus largement et les plus fréquemment répandus dans les manuels scolaires. On pouvait en dire autant de la minorité juive expulsée définitivement d'Espagne en 1492 ;

- une revendication et une revalorisation des dissidents de la politique officielle dans l'histoire contemporaine de l'Espagne et de ceux que l'on avait traités d'hétérodoxes en raison de leur idéologie ou de leur attitude religieuse en des temps proches ou plus lointains, en affirmant en même temps leur caractère espagnol : hérétiques, libéraux, libres penseurs, anarchistes, socialistes, communistes, républicains ; 
- une revalorisation, revendicative elle aussi, des formes historiques de participation à l'exercice du pouvoir, d'autant plus importante que cette participation était en faveur des classes populaires;

- une revendication de la pluralité territoriale et nationale de l'Espagne, de la pluralité des royaumes et des pays différents et différenciés, avec au long des siècles des institutions communes très peu nombreuses mais très puissantes, qui avaient constitué la réalité politique de l'Espagne, situant la formation d'un État authentiquement unitaire à des dates beaucoup plus récentes que ce qui ressortait des manuels scolaires. C'était là un aspect délicat et important: les mouvements, partis et idéologies propres aux différentes nationalités intégrées dans l'État espagnol avaient été durement poursuivis par le régime de Franco, accusés de séparatisme, alors que pendant la Transition, ces nationalités faisaient preuve d'une vigueur renouvelée dans leurs revendications d'autonomie et d'autodétermination ;

- une nouvelle appréciation du peuple plus authentique que celle élaborée et utilisée par le franquisme ;

- une révision critique des impérialismes qui avaient traversé l'histoire de l'Espagne: la longue période de l'Espagne romaine ne fut plus seulement l'intégration positive dans une civilisation plus avancée, mais aussi la fin des cultures autochtones, accompagnée de génocides et d'esclavages en masse.

A ce sujet, il faut mentionner tout spécialement les manières différentes dont le franquisme et la Transition ont parlé de la colonisation hispano-américaine : alors que le premier exaltait les valeurs, positives à ses yeux, du processus et l'interprétait comme l'exemple le plus remarquable de l'accomplissement par l'Espagne de son destin, minimisant ou niant ses aspects négatifs et obscurs, l'histoire enseignée pendant la Transition l'interprétait comme un nouvel exemple du phénomène impérialiste, revendiquant la culture et l'opinion des indigènes soumis.

En ce qui concerne l'histoire universelle, les professeurs révisèrent les personnages, les faits, les processus, les phénomènes déformés ou jugés négativement par le franquisme qui les considérait contraires à son idéologie, sa pratique politique ou à "l'essence de l'Espagne »: la Réforme de Luther, l'Ilustración ${ }^{7}$, les Révolutions française et russe...

Ces nouveautés radicales qui étaient enseignées alors dans quelques classes, cependant de plus en plus nombreuses, ne pouvaient en aucun cas s'appuyer sur les manuels correspondant au programme antérieur à la LGE, mais sur les nouveaux manuels qui étaient publiés année après année au fur et à mesure de la mise en ouvre de la loi. Ces livres étaient eux aussi autorisés par

7 La llustración: mouvement d'idées qui a touché une partie de l'élite socio-culturelle espagnole à la fin du XVIIII siècle, sous l'influence française des "Lumières" (N.D.L.R.). 
le ministère de l'Éducation de Franco. Tout dépendait de l'utilisation et de l'interprétation que chaque professeur leur donnait.

Cette liberté exercée dans les salles de classe par une minorité fut facilitée par un changement qui n'avait rien à voir avec la politique : la disparition de la reválida ${ }^{8}$ permit aux élèves de ne plus recevoir obligatoirement des enseignements uniformes et contrôlés du dehors.

Ces nouveaux contenus, ces nouvelles orientations ne changeaient pas seulement le sens de l'histoire enseignée dans les classes pendant le régime de Franco. Le changement était plus profond : on allait à l'encontre de l'histoire scolaire officielle enseignée depuis plusieurs décennies, une histoire au caractère conservateur, intégriste, déterminée par l'idéologie des groupes sociaux qui avaient détenu le pouvoir politique durant la plus grande partie de l'histoire contemporaine de l'Espagne.

D'où les professeurs avaient-ils tiré de telles connaissances ? Personnellement, j'ai terminé mes études universitaires en 1967 et je peux affirmer que, jusqu'à la fin de cette année-là, le lien avec le régime en place ou la prudence avaient été la ligne de conduite des professeurs universitaires, malgré quelques exceptions, d'ailleurs remarquables.

Il faut chercher la réponse dans cette autre formation que de nombreux étudiants pouvaient trouver en dehors de la classe: séminaires et cercles d'études volontairement formés en marge de l'enseignement officiel, livres achetés dans l'arrière-boutique de quelques librairies dont les propriétaires de plus en plus nombreux osaient prendre des risques, revues spécialisées que l'on commença à publier, voyages à l'étranger que les jeunes universitaires pouvaient se permettre grâce à l'augmentation du niveau de vie de l'Espagne de ces années-là, cours d'été de plus en plus demandés... Bien sûr, les partis et mouvements d'opposition organisés n'étaient pas étrangers à toute cette activité professionnelle.

\section{Écoles différentes, méthodes différentes, didactique différente}

Mais, à mon avis, le changement le plus profond n'était pas celui qui se produisait dans les contenus et les orientations, c'était la nouvelle historiographie qui arrivait dans les salles de classe et dans les méthodes d'enseignement. En effet, l'histoire enseignée à l'époque de Franco consistait en une succession de données, en une narration de faits et une description de phénomènes presque exclusivement politiques. La mémorisation de rois, dynasties, batailles et

8 Reválida : examen que devaient passer dans les lycées officiels tous les élèves des établissements publics et privés à la fin du cycle du bachillerato. 
événements semblait être la substantifique moelle de l'histoire que devaient apprendre les élèves. Les aspects sociaux et culturels fournissaient un complément de moindre importance. L'économie ne figurait que rarement. C'est seulement dans les années soixante que put apparaître de façon très discrète l'influence de l'école des Annales. Cependant, les années de la Transition et les nouveaux manuels entraînèrent dans les classes l'irruption des analyses et interprétations marxistes de l'histoire. Ce changement dans la manière d'organiser la pensée et de mettre les connaissances en application devait être, à terme, plus essentiel que les nouvelles orientations en fonction desquelles étaient traités les grands sujets de l'histoire.

Tout aussi importante fut l'application progressive de nouvelles méthodes d'enseignement et d'une nouvelle didactique de l'histoire. La LGE elle-même luttait pour un enseignement actif. L'apprentissage purement mémorisateur et vide de signification, seul possible jusqu'alors, céda peu à peu la place à un apprentissage plus significatif. L'analyse de textes écrits et d'œuvres artistiques commença à devenir une pratique quotidienne dans les classes, ainsi que l'éveil de l'esprit critique chez les élèves à travers des débats organisés en classe. Et alors que les années soixante et la Transition étaient déjà bien avancées, quelques groupes de professeurs commencèrent même à élaborer des matériels pour que, dans les classes, les élèves puissent apprendre l'histoire selon des procédés proches de la recherche historique ${ }^{9}$. On commença à expérimenter le 102 travail en groupes... Mais ces innovations et ces tentatives intéressantes achoppaient toujours sur le manque de moyens matériels, l'insuffisance des ressources didactiques, l'inadaptation des conditions matérielles des classes et des établissements : obstacles qui entraînaient l'échec et la déformation de la LGE au fur et à mesure qu'elle était implantée. Tout dépendait de la volonté des professeurs, qui manquaient aussi de la formation nécessaire à leurs initiatives.

Ceci explique la désorganisation, les erreurs, les faux pas, la désorientation, le manque de rigueur qui accompagnaient généralement de telles expériences.

La place importante que j'attribue dans cet article à l'activité de cette minorité de professeurs progressistes n'est pas exagérée. Le changement dans les contenus, les orientations, la méthode et la didactique de l'enseignement de l'histoire qu'ils voulaient réaliser dans leurs classes malgré les obstacles et les risques marque une grande différence entre la Transition et l'étape antérieure. D'autre part, l'idéologie et l'activité de ces minorités vont, dans une bonne mesure, orienter la politique éducative des années quatre-vingt, avec l'arrivée au pouvoir du Parti socialiste.

9 II convient de signaler le travail du département d'histoire de l'Institut des sciences de l'éducation de l'université de Valence "Gup Germania - 75 », en raison de la nouveauté qu'il a apportée et de la très large audience qu'il a rencontrée. 


\section{Les années 1980 : la construction de l'État autonomiste}

En 1982, l'arrivée au pouvoir du PSOE, avec la majorité absolue au Parlement, marqua pour tout le monde la fin de la Transition. Dans le domaine particulier de l'enseignement de l'histoire, les thèses de ces minorités qui s'étaient efforcées de changer leur activité dans les classes dès le début de la Transition reçurent un appui officiel. La situation, alors, s'était inversée : à peine restait-il quelques réduits de professeurs qui avaient continué à soutenir les contenus officiels de l'histoire des dernières années du franquisme. Depuis des années les vieux manuels sur lesquels ils pouvaient s'appuyer avaient disparu de la circulation : bon nombre d'entre eux avaient été jugés anticonstitutionnels. D’autre part, les secteurs formés dans des collèges, lycées et universités sous la pure idéologie nationale-catholique étaient totalement minoritaires face à l'arrivée massive de jeunes professeurs. Il ne faut pas oublier que durant la Transition et les premières années de la démocratie, l'Espagne a réalisé le plus grand effort de son histoire en matière de constructions scolaires et, par conséquent, en matière de recrutement de professeurs. Cette minorité nationalecatholique était, en outre, vieillie et proche de la retraite. Le cadre historique était toujours le même : la loi générale d'éducation, corrigée par la Constitution de 1978 .

Dans les classes, les professeurs d'histoire normalisaient leurs orienta-

tions. Les sujets qui marquaient la différence entre dictature et démocratie, entre deux conceptions opposées de l'histoire de l'Espagne, perdirent peu à peu le caractère belligérant qu'ils avaient eu auparavant. De nouvelles générations arrivaient dans les classes, générations dont la manière de vivre n'avait rien à voir avec le franquisme.

Mais le changement politique continuait pourtant en Espagne, dans un cadre maintenant démocratique. Pendant cette décennie, l'Espagne va cesser d'être un État unitaire et centraliste et devenir un État autonomiste. Dans le domaine de l'enseignement, l'État fut divisé en Communautés autonomes ayant les pleines compétences en matière d'éducation (Catalogne, Pays Basque, Andalousie...) et Communautés autonomes qui dépendaient encore du ministère de l'Éducation. Celles qui possédaient les pleines compétences, précisément celles qui se différenciaient le plus culturellement (ayant même leur langue propre, sauf l'Andalousie) introduisirent dans leur cursus des disciplines ou des matières particulières : l'histoire, la culture et, suivant les cas, la langue de la Communauté.

C'est là une des différences en matière de contenus et d'orientations de l'histoire au cours de cette étape politique : la revalorisation, parfois l'exaltation, de la particularité, la différenciation face au territoire voisin. De façon tout à fait fondée, mais parfois, à mon avis, de façon discutable et même abusive. Les 
vieilles questions reviennent alors à l'ordre du jour : qu'est-ce qu'une culture? qu'est-ce qu'une nation?

Par ailleurs, un événement essentiel dans l'histoire de l'Espagne s'est produit récemment sans qu'on lui accorde la place qu'il mérite dans l'enseignement de l'histoire : l'entrée de l'Espagne dans la Communauté européenne. Mes affirmations ne peuvent être fondées que sur mes observations personnelles, non sur un travail de recherche. Cet événement si remarquable apparaît dans les manuels, sans toutefois l'importance ni la profondeur nécessaires; de plus, l'utilisation que les professeurs font de ces manuels tend à le minimiser.

Cependant, la grande différence entre cette décennie et la précédente va porter sur les façons d'enseigner, sur la méthodologie et la didactique de l'histoire, plutôt que sur les contenus. La démocratisation de l'enseignement n'entraîne pas seulement une nouvelle organisation scolaire et une nouvelle orientation des connaissances, mais aussi une nouvelle façon de les acquérir et de les utiliser. Le but maintenant est que l'élève se transforme de sujet passif de l'enseignement en sujet actif pour construire son propre apprentissage. Dans la discipline de l'histoire, le manuel et les explications du professeur laissent aux élèves du temps pour élaborer de petits travaux d'enquête et de recherche, la dynamique traditionnelle de la classe se transforme avec le travail de groupe ; la mise en scène de faits ou de phénomènes historiques essaie de susciter l'attention des élèves et d'éveiller leur intérêt, les moyens audiovisuels cessent d'être 104 quelque chose d'exceptionnel... Mais tous ces changements réclament une nouvelle formation des professeurs et une augmentation substantielle des moyens financiers consacrées à l'enseignement. Car il est vrai que c'est encore une minorité de professeurs qui teste, expérimente, propose et ouvre de nouvelles voies.

On essaie de répondre à la première demande par l'ouverture des centres de professeurs, créés pour encourager, appuyer et soutenir l'activité des nombreux groupes de travail qui apparaissent, et aussi pour favoriser et canaliser la formation continue des professeurs. Il n'est pas exagéré d'affirmer que dans cette effervescence professionnelle les professeurs d'histoire ont joué un rôle important. Mais la deuxième demande n'a pas été satisfaite: les moyens destinés à l'enseignement sont restés très en dessous des besoins créés par les nouvelles attentes.

\section{L'actualité}

Même si cela déborde le sujet de cet article, le grand débat qui s'est ouvert en Espagne sur l'enseignement de l'histoire mérite bien qu'on lui consacre quelques lignes. Ce débat est centré autour d'une seule question: quelle histoire enseigner?

La mise en place de la loi d'aménagement général du système éducatif approuvée en 1990 remplace peu à peu le système établi par la loi générale 
d'éducation. La LOGSE instaure l'enseignement obligatoire jusqu'à seize ans en créant un enseignement secondaire obligatoire du type tronc commun, pratiquement identique pour tous. C'est ainsi que l'on a pris conscience, avec plus de netteté encore qu'auparavant, des sérieuses difficultés de l'adolescent moyen pour comprendre les contenus de l'histoire telle qu'elle est enseignée. D'autre part, le fait que dans le cycle du bachillerato, d'une durée de deux ans, l'on ne traite pratiquement que l'histoire contemporaine a déjà été remis en question.

La difficulté de compréhension des élèves dépend-elle de la difficulté des contenus ou de l'inadéquation des méthodes et de la didactique? Faut-il changer les contenus? L'importance concédée à la période contemporaine entraînera-t-elle dans l'avenir la dévalorisation de nos racines historiques et culturelles?

Bien évidemment, aucune autre discipline n'est aussi dépendante des avatars politiques.

$$
\text { *** }
$$

En conclusion, trois éléments de cette analyse doivent être soulignés. En premier lieu, l'enseignement de l'histoire en Espagne a connu un changement considérable pendant la Transition politique, dans les années soixante. Instrument d'endoctrinement et de justification durant le franquisme, elle est devenue un instrument de délégitimisation de ce même régime. A la fin de la Transition, l'enseignement de l'histoire présente dans ses objectifs, ses contenus et ses orientations des caractéristiques semblables à celles de tout autre pays d'Europe occidentale et rencontre les mêmes problèmes.

Second point, le changement d'orientation qui s'est produit dans cette discipline pendant la Transition a eu deux agents: de nouveaux manuels scolaires, publiés par des auteurs qui s'inspiraient des courants historiographiques des Annales et des marxistes, et une minorité croissante de professeurs, généralement autodidactes, qui arrivaient dans les classes avec des idées nouvelles et le désir d'en finir avec la dictature.

Enfin, ce processus de changement fut involontairement favorisé par le nouveau cadre législatif instauré par la loi générale d'Éducation, approuvée et implantée dans les dernières années de la dictature. 